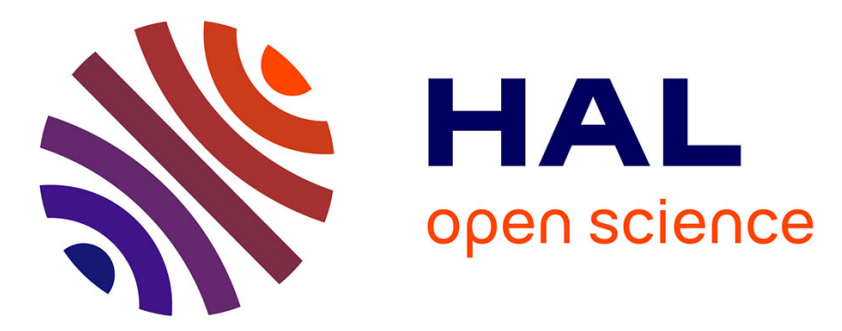

\title{
Molecular insights into mechanisms of GPCR hijacking by Staphylococcus aureus
}

Claire Grison, Paul Lambey, Sylvain Jeannot, Elise del Nero, Simon Fontanel, Fanny Peysson, Joyce Heuninck, Rémy Sounier, Thierry Durroux, Cedric Leyrat, et al.

\section{To cite this version:}

Claire Grison, Paul Lambey, Sylvain Jeannot, Elise del Nero, Simon Fontanel, et al.. Molecular insights into mechanisms of GPCR hijacking by Staphylococcus aureus. Proceedings of the National Academy of Sciences of the United States of America, 2021, 118 (42), pp.e2108856118. 10.1073/pnas.2108856118. hal-03426718

\section{HAL Id: hal-03426718 https://hal.science/hal-03426718}

Submitted on 12 Nov 2021

HAL is a multi-disciplinary open access archive for the deposit and dissemination of scientific research documents, whether they are published or not. The documents may come from teaching and research institutions in France or abroad, or from public or private research centers.
L'archive ouverte pluridisciplinaire HAL, est destinée au dépôt et à la diffusion de documents scientifiques de niveau recherche, publiés ou non, émanant des établissements d'enseignement et de recherche français ou étrangers, des laboratoires publics ou privés. 


\section{Main Manuscript for}

Molecular insights into mechanisms of GPCR hijacking by Staphylococcus aureus

Claire M. Grison ${ }^{1}$, Paul Lambey ${ }^{1}$, Sylvain Jeannot ${ }^{1}$, Elise Del Nero ${ }^{1}$, Simon Fontanel ${ }^{1}$, Fanny Peysson ${ }^{1}$, Joyce Heuninck ${ }^{1}$, Rémy Sounier ${ }^{1}$, Thierry Durroux ${ }^{1}$, Cédric Leyrat ${ }^{1}$, Sébastien Granier $^{1 *}$ and Cherine Bechara $^{1,2, *}$

${ }^{1}$ IGF, University of Montpellier, CNRS, INSERM, Montpellier, France

${ }^{2}$ Institut Universitaire de France (IUF), Paris, France

*Corresponding authors S. Granier and C. Bechara

Email: sebastien.granier@igf.cnrs.fr and cherine.bechara@igf.cnrs.fr

\section{Author contributions:}

CG, SG and CB designed the research. CG, CL, PL, SJ, SF, FP and RS designed, expressed and purified the proteins. CG and JH performed and analysed cell-based assays with the help of TD and CB. EDN performed and analysed HDX data with CB. CL performed modelling and computational analysis of ACKR1. CB performed and analysed native MS experiments. CB analysed the data, made the figures and wrote the manuscript with the help of SG and contributions from all authors. SG and CB supervised the research.

\section{Competing interests:}

The authors declare no competing interests.

Classification: Biological Sciences, Biochemistry

Keywords: GPCR, host-pathogen interactions, structural mass spectrometry, pharmacology.

\section{This PDF file includes:}

Main Text 


\begin{abstract}
Atypical chemokine receptor 1 (ACKR1) is a $\mathrm{G}$ protein-coupled receptor (GPCR) targeted by Staphylococcus aureus bi-component pore-forming leukotoxins to promote bacterial growth and immune evasion. Here we have developed an integrative molecular pharmacology and structural biology approach in order to characterize the effect of leukotoxins HlgA and HlgB on ACKR1 structure and function. Interestingly, using cell-based assays and native mass spectrometry, we found that both components HlgA and $\mathrm{HlgB}$ compete with endogenous chemokines through a direct binding with the extracellular domain of ACKR1. Unexpectedly, HDX-MS analysis revealed that toxin binding allosterically modulates the intracellular $\mathrm{G}$ protein-binding domain of the receptor, resulting in dissociation and/or changes in the architecture of ACKR1-Gail protein complexes observed in living cells. Altogether, our study brings important molecular insights into the initial steps of leukotoxins targeting a host GPCR.
\end{abstract}




\section{Significance statement:}

Bi-component leukotoxins represent a promiscuous group among the virulence factors secreted by $S$. aureus that helps the pathogen to develop and evade host immune system. These toxins specifically recognize host GPCRs in an unknown molecular mechanism and functional effects on receptor's biology. In this work, using the $\gamma$-hemolysins $\mathrm{HlgA}$ and $\mathrm{HlgB}$, we identify ACKR1 as a receptor for $\mathrm{HlgB}$ and demonstrate that toxins compete with ACKR1 natural ligand. Furthermore, we find that binding of leukotoxins leads to an allosteric conformational change in ACKR1 intracellular part and interferes with receptor architecture, a process that was correlated with a destabilisation of receptor-G protein interaction

in living cells. Overall, our findings lay the ground for the development of antimicrobials by shielding leukotoxin-GPCRs interactions. 


\section{Introduction}

Staphylococcus aureus (SA) is a major concern for public health mainly due to the emergence of various multidrug-resistant strains of this pathogen $(1,2)$. SA produces a large arsenal of virulence factors, among which bi-component leukocidins - also referred to as leukotoxins - stand out as interesting targets for developing novel antivirulence strategies $(3,4)$. Leukotoxins belong to the family of $\beta$-barrel pore-forming toxins that assemble into heteromeric pores to lyse specific cells (5-8). Five different types of leukotoxins are expressed by SA infecting humans: PVL, LukED, HlgAB, HlgCB and LukAB. Each one is formed by two subunits, the host cell targeting $\mathrm{S}$ component ( $\mathrm{S}$ for slow elution during biochemical purification) and the polymerization $\mathrm{F}$ (fast elution) component. In the current view, with the exception of LukAB, all the subunits are believed to be secreted as monomers that will heterodimerize upon specific interaction with host myeloid and erythroid cells. Dimerization will lead to a subsequent toxin oligomerization and poreformation in cell membranes (9-11).

Recent identification of leukotoxins receptors in targeted host cells increased our knowledge of the mechanism behind cellular specificity of these toxins and their role in pathogenesis (12-17). Based on these findings, the predicted mechanism is that only the monomeric $\mathrm{S}$ component specifically interacts with various complement and chemokine receptors present on the surface of leukocytes, all related to the family of G protein-coupled receptors (GPCRs). The S-component later recruits the F-component to trigger oligomerization and pore formation. Though the F-components HlgB and LukD where shown to bind to the surface of erythrocytes independent from their S-component partners $(18,19)$, it was considered a receptor-independent binding. Recently, one of the F-components, LukF-PV, was shown to specifically require a receptor in order to recognize targeted cells (20), challenging therefore the proposed initial steps of receptor recognition and pore formation (21).

Out of all targeted receptors, the atypical chemokine receptor 1 (ACKR1, previously called DARC) (22) recognised by both $\mathrm{HlgA}$ and LukE is a key player. Indeed, in addition to being expressed in myeloid cells, ACKR1 is expressed in erythrocytes and endothelial cells making it necessary for SA to escape our immune system, to grow and to cause cell death $(12,13)$. Unlike canonical chemokine receptors, ACKR1 lacks the conserved DRYLAIV motif and is thus structurally unable to activate G proteins by dissociating the subunits upon chemokine engagement $(23,24)$. Rather, it internalizes and transports chemokines to the degradative compartment, acting as a chemokine buffer by modulating chemokine concentration and 
bioavailability (25-28). The high-resolution structure of ACKR1 is still unknown, however its homologues of known structures share the highly conserved GPCR structure, consisting of a single polypeptide chain with three intracellular and extracellular loops, an external N-terminal region essential for the specificity of ligand binding, and an intracellular $\mathrm{C}$-terminal region that is involved in receptor signalling. Although increasing amount of structural and molecular data of chemokine receptors are being discovered $(29,30)$, the structural immunology and pharmacology related to ACKR1 is still in its infancy.

Binding of leukotoxins to GPCRs is poorly understood at the molecular and structural level. Various residues in the loops of the rim domain of HlgA and LukE as well as a 4-residue region in the cap domain of HlgA were shown to be necessary for the haemolytic activity and/or binding to erythrocytes (31-33). From the receptor side, LukE and HlgA seem to target different regions of ACKR1 N-terminal part, a highly flexible region, whereas both require sulfation of tyrosine residues in this same part of the receptor (13). In addition, little is known on the effects of leukotoxins on ACKR1 binding to its natural ligands and downstream molecular signalling. Although biochemical and cell biology work has been done since the discovery of receptors targeted by leukotoxins, direct evidence capturing purified leukotoxin-receptor complexes has only been provided for the LukE-CCR5 pair (16).

In this study, we used an integrative molecular pharmacology and structural mass spectrometry (MS) approach in order to characterize the effect of HlgAB binding on ACKR1 structure and function. We demonstrate that both leukotoxins, $\mathrm{HlgA}$ and $\mathrm{HlgB}$, form independent complexes with purified ACKR1 in vitro using native MS (nMS). In living cells, TR-FRET experiments revealed that both HIgA and HlgB binding to ACKR1 compete with CCL5, an endogenous ligand. We also monitored the effect of leukotoxin binding on ACKR1 conformation using a combination of Hydrogen/Deuterium exchange-MS (HDX-MS) and cell-based resonance energy transfer. Surprisingly, in addition to the expected accessibility changes in the extracellular domain of the receptor, binding of leukotoxins induced long-range allosteric conformational changes in the intracellular domain of ACKR1 that leads to the dissociation and/or changes in the architecture of preassembled ACKR1-Gail protein complexes. Altogether, our study brings novel insights into the initial steps of leukotoxins biology through GPCR, namely the toxins effect on GPCR structure and function. 


\section{Results}

\section{nMS reveals $\mathrm{HlgB}$ and $\mathrm{HlgA}$ dimers in solution with $\mathrm{HlgB}$ being more prone to dimerization}

We first analysed purified recombinant HlgA and HlgB by nMS in order to verify their oligomeric state, in the absence and the presence of detergent micelles as a mimic of the amphiphilic membrane environment. nMS has been gaining ground and has become a key actor in studying membrane protein interactions and dynamics (34-39). It preserves non-covalent interactions and gives information regarding the stoichiometry and binding partners of protein complexes, among which GPCRs $(40,41)$.

Recombinant $\mathrm{HlgA}$ and $\mathrm{HlgB}$ were present mainly as monomers in vitro, however we also detected some dimeric species (Fig. 1A and B, Fig S1A) even at the lowest concentration analysed (sub- $5 \mu \mathrm{M}$ ). In the presence of detergent, $\mathrm{HlgA}$ and $\mathrm{HlgB}$ were able to bind multiple DDM molecules in their monomeric and dimeric forms (Fig. S1B), and detergent molecules were easily dissociated upon higher activation in the gas phase. An equimolar mixture of $\mathrm{HlgA}$ and $\mathrm{HlgB}$ resulted in the formation of heterodimers in vitro, in the absence of any receptor (Fig. 1C). In all analysed conditions, HlgB was systematically more prone to dimerization than $\mathrm{Hlg} \mathrm{A}$, since the dimer-to-monomer ratios at a given concentration was always 2-fold times higher for $\mathrm{HlgB}$ compared to HlgA. Taken together, our results demonstrate the presence of toxin homo- and hetero-dimers in solution even in the absence of membranes and receptors, HlgB being more prone to dimerization compared to HlgA.

\section{Purification of homogeneous human atypical chemokine receptor ACKR1}

The GPCR ACKR1 was first produced in Sf9 cells and purified in DDM detergent, which gave a homogeneous monodisperse main peak by size-exclusion chromatography, as well as an additional smaller peak eluting at a higher apparent mass (Fig. 1D). nMS analysis of both peaks shows the presence of both monomeric and homodimeric ACKR1, however with additional heterogeneous masses around 3.5-5 kDa per monomer (Fig. S2A). We hypothesised that the nature of the observed additional mass came from N-glycosylations. In order to confirm our hypothesis, we produced ACKR1 in a cell line with restricted and homogeneous $\mathrm{N}$-glycosylations ( $\mathrm{HEK} \mathrm{GnTI}^{-}$), which decreased the heterogeneity but did not lead to the complete removal of ACKR1 modifications (Fig. 1E, F and Fig. S2B). Finally, treatment of purified receptor with PNGaseF resulted in a complete removal of the modification, implicating that they indeed are $\mathrm{N}$-glycosylations (Fig. S2C). We then verified all reported potential N-glycosylation sites by point mutations (Fig. S2D to F) and found that all three N16, N27 and N33 are glycosylated in ACKR1. 


\section{HlgB and HlgA both bind separately to monomeric ACKR1 but not concomitantly}

In order to provide a direct evidence of ACKR1-leukotoxin binding and determine the stoichiometry of this complex, we analysed mixtures of purified recombinant HlgA, HlgB and ACKR1 using nMS. The low yield of dimeric ACKR1 lead us to perform in vitro experiments only with the monomeric form. Incubating ACKR1: HlgA in a 2:1 ratio, ACKR1 being used at higher concentration to overcome lower ionization efficiency, for $30 \mathrm{~min}$ at $4^{\circ} \mathrm{C}$ revealed the presence of $\mathrm{m} / \mathrm{z}$ species with a mass corresponding to proteins alone, as well as the presence of an ACKR1-HIgA complex with a 1 to 1 stoichiometry (Fig. 2A). Binding of HlgA gave similar results with WT and deglycosylated ACKR1, with peaks better defined in the mass spectra in the latter case. These results thus evidence the existence of GPCR-leukotoxin complex in detergent micelles and suggest that receptor glycosylation is not strictly necessary for ACKR1-HlgA interaction to occur. Surprisingly, we detected the formation of ACKR1-HlgB complexes when mixing the F-component $\mathrm{HlgB}$ with the receptor (Fig. S3), demonstrating a specific interaction between $\mathrm{HlgB}$ and ACKR1. Finally, when mixing the three components ACKR1: HlgA: HlgB in a 2: 1: 1 ratio, we detected separately formed ACKR1-HlgA and ACKR1-HlgB complexes, but no ternary ACKR1-HlgA-HlgB complexes were visible. ACKR1-leukotoxins complexes were not stable enough to overcome a size-exclusion chromatography (SEC) step since receptor-leukotoxin mixtures did not yield a stable complex peak upon SEC. This points to a low-affinity binding observed in vitro, and could also explain the low relative intensity of the complexes observed by nMS. Taken together our nMS results capture the first leukotoxin-GPCR complex in solution in a purified system and demonstrate that the Fcomponent $\mathrm{HlgB}$ also interacts directly with ACKR1.

\section{Both leukotoxins compete with ACKR1 natural ligand and HIgB binding is cooperative}

In order to validate the observed binding in vitro and to assess the effect of $\mathrm{HlgA}$ and $\mathrm{HlgB}$ on ACKR1 binding to an endogenous ligand (CCL5) (28), we carried out competitive binding assays in living HEK293T cells by Homogenous Time Resolved FRET (TR-FRET) technology (42) (Fig. 2B). SNAPtag-fused ACKR1 receptor (ST-ACKR1) transiently expressed in HEK293 cells was covalently labelled with Lumi4-terbium as donor. Cells were then incubated in the presence of d2-CCL5, a fluorescent derivative of CCL5 used as ligand tracer. After assay validation (Fig. S4A, B), HlgA and HlgB binding was assessed by competition experiments (Fig 2B). The results revealed that both leukotoxins compete with d2-CCL5 with a slightly higher displacement in the case of $\mathrm{HlgB}$ (IC50 $=577 \pm 93$ and 376 $\pm 105 \mathrm{nM}$ respectively), confirming the capacity of both toxins to bind to ACKR1 as seen by nMS. Surprisingly, the 
slopes of the competition curves are consistently different, close to -1 for $\mathrm{HlgA}$ and close to -2 for $\mathrm{HlgB}$, suggesting the existence of a positive cooperative binding process with HlgB. This could be correlated with the higher ability of HlgB to homo-dimerize compared to HlgA as observed by nMS with purified toxins. Finally, binding affinity of an equimolar $\mathrm{HlgA}$ and $\mathrm{HlgB}$ mixture resulted in a dramatic left shift of the IC50 of the competition curve $(6.2 \pm 2.7 \mathrm{nM})$ and the slope of the curve, as observed with $\mathrm{HlgB}$, presented the characteristics of a positive cooperative binding process (Fig. 2B). The observed increased

affinity and cooperative binding might originate from an increased avidity, similar to what is observed with bivalent ligands (43). Indeed, nMS showed that HlgB is more prone to homo-dimerize compared to $\mathrm{HlgA}$, which may explain the cooperativity observed only for HlgB and the lower IC50 for the latter, whereas a mixture of HlgA and HlgB will lead to hetero-oligomerization with the potential formation of octamers forming the (pre-)pore at the vicinity of the membrane, resulting in an enhanced affinity through avidity.

\section{Conformational changes of ACKR1 upon binding to leukotoxins revealed by HDX-MS}

In order to determine potential conformational changes occurring in ACKR1 upon binding to leukotoxins, we developed an HDX-MS strategy using purified ACKR1 and leukotoxins in vitro following recommendations (44). Since purification mainly yield monomeric ACKR1 and low amounts of dimers in detergent micelles (Fig. 1D), we focused our attention on monomeric ACKR1. GPCRs are known to maintain their functional state as monomers $(45,46)$, therefore we anticipated that studying the effect of leukotoxins binding to ACKR1 monomers may provide relevant information to decipher the mechanism by which the toxins modify ACKR1 function.

HDX-MS gives information related to solvent accessibility and dynamics of biological complexes. It is based on the exchange kinetics between Deuterium atoms (D) present in the buffer and amide protons $(\mathrm{H})$ of native polypeptide chains in solution (47). Unlike higher resolution approaches, HDX-MS offers the advantage of obtaining dynamic structural information for samples that presents heterogeneous and/or flexible areas. It was successfully applied to characterize the dynamics and interactions of membrane transporters and even GPCRs (48-53).

HDX-MS optimization for apo ACKR1 allowed the identification of 77 peptides covering $70.1 \%$ of WT ACKR1, with 3.41 peptide redundancy (Fig. S5A). The highly glycosylated N-ter part of ACKR1 was mainly missing, therefore we performed the same experiment with the non-glycosylated $\mathrm{N}^{16,27,33} \mathrm{Q}$ ACKR1 mutant which resulted in an increased sequence coverage at the N-ter level (Fig. S5B). In order 
to determine the effect of $\mathrm{Hlg} \mathrm{A}$ and $\mathrm{HlgB}$ binding on ACKR1 conformational dynamics, we performed differential HDX ( $\triangle \mathrm{HDX})$ analysis between apo ACKR1 and equimolar mixtures of ACKR1: HlgA or HlgB. Biological replicates (2 with WT ACKR1 and 1 with $\mathrm{N}^{16,27,33} \mathrm{Q}-\mathrm{ACKR} 1$ ) were freshly prepared, and deuteration timepoints were performed in triplicates for each condition (HDX summary table, data S1). The total number of usable ACKR1 peptides in the presence of leukotoxins were lower compared to the receptor analysed alone due to the overlap with the peptides coming from leukotoxins, which are soluble proteins of roughly the same size of the receptor. This resulted in a decreased overall redundancy (HDX summary table, data S1).

In order to visualize the leukotoxin-induced changes on ACKR1 and in the absence of a high-resolution structure of the latter, we generated a model for ACKR1 that was validated against HDX-MS data using molecular dynamics simulations (MDS) (Fig. S6, see Methods). Both leukotoxins had overall similar structural effects on purified monomeric ACKR1, which correlates with the similar binding affinities measured in living cells by TR-FRET. ACKR1 long N-terminal part was mainly protected in the presence of leukotoxins (peptide 9-20 more protected compared to peptide 27-45, the latter only visible in the deglycosylated sample), whereas HDX data for the majority of ECLs was not present due to the missing sequence coverage (Fig. 3, Fig. S5 C-G). The N-terminal part, containing a sulfotyrosin Y41 residue reported as highly important for ACKR1-mediated pore-formation by $\mathrm{HlgAB}$ (13), relates therefore to the direct binding site of leukotoxins. The overall deuteration of detected TM segments was very low, most likely due to the presence of the detergent micelles surrounding these parts that shield them from the solvent. However, the upper part of H5 (residue 203-215) presented a higher uptake compared to the other TM, and was slightly more protected in the presence of the toxins compared to the receptor alone (Fig. 3, Fig. S5 C-G). This region also displays higher flexibility in MDS of generated ACKR1 model (Fig. S6). The upper part of TM5 was shown to be important for various CKR-ligand interactions (30), whereas rearrangements in TM5 was shown to play critical roles in signal transmission for various GPCRs (54). Therefore, this region can be directly implicated in the leukotoxin binding to ACKR1. Strikingly, $\triangle H D X$ shows that binding of leukotoxins induced allosteric conformational changes that lead to the protection of the H8 and the C-terminal part of ACKR1, as well as ICL2 and to a lower extent parts of ICL1 (Fig. 3, Fig. S5 C-G), domains that are known to be critical for Ga binding (55). The change in the C-terminal 330-339 peptide should be interpreted with caution. Indeed, this domain is most likely unstructured and exploring multiple conformations in detergent micelles. One possible interpretation could be that this part becomes less flexible and might make some contacts with the intracellular domain of ACKR1 upon 
binding to the toxins. Additional studies will however be required to validate this hypothesis. On the other hand, $\triangle \mathrm{HDX}$ results for $\mathrm{H} 8$ suggests that this helix might change its conformation to shield both ICL1 and ICL2 upon binding of leukotoxins to ACKR1, in a way that blocks G protein accessibility similar to what was observed for Angiotensin 2 receptor AT2R (56). The structural dynamics of H8 is indeed suggested to play an important role in GPCR signalling (57).

\section{HlgB and HlgAB interfere with preassembled ACKR1-Goi complexes in living cells}

The surprising allosteric modulation observed by HDX prompted us to analyse the ACKR1-Gi interactions in living cells. We did not see any G protein activation with ACKR1 in the presence of chemokines CCL2 and CCL5, as demonstrated by the absence of a BRET signal decrease between $\alpha$ and $\beta \gamma$ subunits of the studied G proteins (Fig. S7A, B). This is not surprising for ACKR1 since it lacks the canonical DRY motif involved in G protein activation (22). However, when we followed the direct interaction between the C-terminal part of ACKR1 and the Gai1 subunit by BRET (Fig. 4A), we observed a constitutive BRET signal in the absence of any ligand, indicating the existence of ACKR1-YFP- Gai1RLuc complexes in basal conditions (Fig. S7C, F). This could be similar to the decoy action ACKRs have regarding chemokines present in the extracellular milieu, wherein ACKR1 could also help regulating the concentration of Gai1 in the intracellular milieu. Measured BRET signal from ACKR1-Gai1 interaction followed a saturation curve, unlike the BRET measured between ACKR1 and another G $\alpha$ protein, Gaq, which gave a non-specific linear signal (Fig. S7D). Interaction between ACKR1 and Gai1 was further validated in vitro by a pull-down experiment using both purified proteins (Fig. S7E).

Adding up to $10 \mu \mathrm{M}$ of CCL5 or HlgA promoted a weak variation of the BRET signal between ACKR1YFP and Gai1-RLuc, whereas incubation of cells with increasing concentrations of HlgB or HlgAB strongly reduced basal BRET signal (Fig 4B), the effect of HlgAB being nearly 10-fold more potent compared to the effect of $\mathrm{HlgB}$ (EC50 $796 \pm 110 \mathrm{nM}$ and $14 \pm 3 \mathrm{nM}$ for HlgB and HlgAB respectively). This suggests a conformational rearrangement that modifies the distances and/or orientation within the ACKR1-YFP-Gai1-RLuc complexes in the presence of HlgAB and to a lesser extent HlgB. To further validate the specificity of the observed effect, we performed the same experiments using the mutant Y41FACKR1. When mutating the sulfotyrosin Y41, reported as highly important for ACKR1-mediated poreformation by $\mathrm{HlgAB}$ (13), we observed a 10-fold decrease in the effect of HlgAB (Fig. 4C). Interestingly, mutating Y30, another potentially sulfated tyrosine present at the Nter, did not result in any significant 
effect on ACKR1-Gai1 binding, implying that this site is either not sulfated or that sulfation of this tyrosine is not important for binding to leukotoxins, as reported previously (13). These results thus demonstrate that ACKR1-Goil architecture can be modified in living cells upon leukotoxins binding, confirming the biological relevance of the allosteric modifications observed by HDX-MS. Intriguingly, however, HlgA was not able to recapitulate the effect observed with $\mathrm{HlgB}$, suggesting that additional conformational rearrangements are involved in the observed effects on ACKR1-Gail complexes.

\section{HlgAB and HlgB interfere with ACKR1-ACKR1 interactions in living cells}

To address the question of additional ACKR1 receptor conformational changes, based on the observed positive cooperativity for $\mathrm{Hlg} \mathrm{AB}$ and $\mathrm{HlgB}$ but not $\mathrm{HlgA}$ binding and given that GPCRs are notoriously known to form oligomers including in native tissues (58), we suspected that the toxins binding could modify receptor oligomerization. In order to explore these mechanisms, we monitored the effects of leukotoxins on receptor-receptor interactions in living cells using two different resonance energy transfer (RET) strategies: lanthanide-based TR-FRET and bioluminescent RET (BRET). BRET is based on the fusion of an energy donor (RLuc or NanoLuc) and an energy acceptor (YFP) to the C-terminus of ACKR1 (59) (Fig. 5A), whereas TR-FRET is based on the fusion of a SNAP-tag to ACKR1 extracellular Nterminus that will be labeled with a FRET donor and an acceptor (60) (Fig. 5B). In both techniques, RET signal is sensitive to the distance between the donor and the acceptor while in BRET it is more sensitive to the relative orientation of the fluorescent probes. Indeed, the error in distances measured via lanthanidebased RET due to the orientation factor was shown to be essentially negligible (61). The different positioning of the probes can thus sense various receptor rearrangements both in the extracellular and intracellular domains of this GPCR.

We first confirmed the propensity of ACKR1 to form oligomers in living cells using both RET approaches. Indeed, saturation of the BRET signal when increasing ACKR1-YFP expression while ACKR1-Nanoluc remained constant strongly supports receptor oligomerization (Fig. S7F). Though BRET assessment of oligomerization can be notoriously difficult (62), our results are in agreement with a previous report that describes the presence of homo-oligomeric ACKR1 by BRET (63). Similarly, a constitutive TR-FRET signal was recorded when N-ter SNAP-tagged ACKR1 was expressed and labelled with both Lumi4-Tb (donor) and d2 (acceptor) (Fig. 5B). Altogether, BRET and TR-FRET results correlate with the presence of purified stable ACKR1 dimers in solution detected by nMS (Fig. 1E), confirming the capacity of ACKR1 to oligomerize. 
Using the experimental conditions corresponding to the BRET50, we evaluated the impact of HlgA and $\mathrm{HlgB}$ binding on BRET signal. HlgA did not induce any modification in the BRET signal while HlgB induced a BRET signal increase (Fig. 5A). Interestingly, HlgAB equimolar mixture resulted in an increased BRET signal, with a tenfold higher potency compared to $\mathrm{HlgB}(18.6 \pm 2.5 \mathrm{nM}$ versus $178 \pm 28 \mathrm{nM}$ ). The increased BRET signal can be due to an increase in oligomer density and/or to a change in the architecture of Cter part of ACKR1 leading to a closer proximity and/or a reorientation of the probes, in the presence of $\mathrm{Hlg} \mathrm{AB}$ and $\mathrm{HlgB}$, but not with $\mathrm{HlgA}$ at the tested ligand concentrations. Remarkably, this correlates with the conditions showing a cooperative effect in competitive binding assays (Fig. 2B). In contrast to the BRET signal increase, the data revealed a decrease in TR-FRET signal between ACKR1 receptors in the presence of $\mathrm{HlgB}$ and $\mathrm{Hlg} \mathrm{AB}$ while no significant effect was observed with $\mathrm{HlgA}$ at the tested ligand concentrations. Again, the potency of $\mathrm{HlgB}$ was 10-fold lower compared to HlgAB $(429 \pm 138$ versus $47 \pm 15 \mathrm{nM})$ in the same range of the BRET assays. The one log difference between $\mathrm{HlgB}$ and HlgAB observed in both BRET and TR-FRET is similar to the difference observed in competitive binding experiments (Fig. 2B). Taken together, the observation of BRET signal increase and TR-FRET signal decrease strongly suggest that $\mathrm{HlgB}$ and $\mathrm{HlgAB}$ induce large conformational changes leading to a rearrangement of the oligomeric architecture that positions the fluorescent probes further apart in the N-termini and closer together and/or with a different orientation in the C-termini (Fig. 6). These conformational changes in oligomeric assemblies may also explain the observed rearrangement in ACKR1-Gail complexes. 


\section{Discussion}

SA leukotoxins targeting GPCRs represents an attractive aspect in modulating GPCR function and remains largely unexplored. We chose to focus on ACKR1 since it is a crucial target for SA pathogenesis, being not only expressed in myeloid cells like the other targeted receptors, but also in erythrocytes and endothelial cells. We demonstrate that HlgB also recognises ACKR1, making it the second F-component leukotoxin with an identified receptor. Our results may explain the observation that HlgB binds to erythrocytes independently from HlgA (18). Unlike previously thought (17), our data demonstrate that both HlgA and HlgB separately bind host ACKR1 during the initial steps to initiate pore formation. Both leukotoxins were able to compete with ACKR1 natural ligand, however with a difference in the mechanism. The cooperativity that accompanies toxin ability to dimerize and oligomerize seems a key factor that drives the effects observed on ACKR1 conformational changes in living cells. While HlgB shows a higher ability to homodimerize compared to HlgA, the presence of both $\mathrm{HlgA}$ and $\mathrm{HlgB}$ at the vicinity of a membrane expressing their targeted receptor induces the formation of an octameric pore (9, 10). Dimerization and oligomerization increase the avidity in the system which in turns increases the effects of $\mathrm{HlgBB}$ and $(\mathrm{Hlg} \mathrm{AB})_{4}$ on ACKR1.

Our data also strongly suggests that the capacity of $\mathrm{HlgB}$ and $\mathrm{H} \lg \mathrm{AB}$ to dimerize will lead to changes in ACKR1-ACKR1 constitutive interactions in living cells that may also impact receptor function. Unfortunately, we were not able to address conformational changes in ACKR1 dimers due the very low yield of stable purified dimers obtained upon purification. However, HDX-MS with monomeric ACKR1 revealed quite unexpected conformational changes that may participate, at least in part, to the conformational rearrangement of oligomers observed in living cells. Indeed, toxins binding to the monomeric receptor is not neutral and induces conformational changes in the extracellular N-terminal part, which is directly involved in leukotoxins binding, and more surprisingly in the C-terminal part at the level of H8. This allosteric long-range modulation thus propagates to the G protein binding sites in the ICLs (Fig. 6), correlating with the BRET assays we used to follow ACKR1-Gai1 interactions in living cells. While $\mathrm{HIgB}$ and $\mathrm{Hlg} \mathrm{AB}$ binding destabilizes ACKR1-Gai1 protein complexes, which could release available Gai1 protein in the intracellular milieu, HlgA binding does not interfere with ACKR1-Gai1 protein complexes. Indeed, though $\mathrm{HlgA}$ is able to bind and induce conformational changes in ACKR1 monomer, this process is apparently not sufficient to modify the oligomeric architecture of ACKR1 as demonstrated by the TR-FRET and BRET assays, probably due a lower propensity of this toxin component 
to form oligomers. Further studies will be needed to determine whether this phenomenon has functional implications in the pathogenicity of the SA through modulation of $\mathrm{G}$ protein and related signaling pathways.

Only the presence of $\mathrm{Hlg} \mathrm{AB}$ heterodimer can lead to pore-formation (18), but the structural and molecular mechanisms of this process remain to be understood. Further studies manipulating the oligomerization of toxins and ACKR1 (in proteoliposomes for instance) will thus be important to shed the light on the mechanisms behind conformational changes of both leukotoxins and the potential role of the GPCR receptor during the different pore-formation steps. Notwithstanding, our findings may open the way to develop antibiotics inhibiting the first and limiting step of toxin action by targeting host receptors binding, an inhibition that has the potential to be less prone to resistance. 


\section{Materials and Methods}

Protein constructs. For production in insect or human cells, the full-length synthetic gene of human ACKR1 isoform-2 (UniprotKB-Q16570) was subcloned into modified pFastBac or pCMV-Dest vectors (Thermofischer) respectively, resulting in the full-length ACKR1 bearing the influenza virus hemagglutinin (HA) signal peptide followed by a Flag epitope at N-terminal. For human cell-based assays, the full-length synthetic gene of human ACKR1 was subcloned into pcDNA3.1-YFP vector in frame with the N-terminus of YFP resulting in ACKR1-YFP, into pcDNA3.1-Nanoluc vector in frame with the Nterminus of Nanoluc resulting in ACKR1-Nanoluc, and into pcDNA3.1-SNAP vector in frame with the C-terminus of SNAP tag resulting in SNAP-ACKR1. The synthetic genes of mature Staphylococcus aureus HlgA (UniprotKB-P0A074) residue 30-309 and HlgB (UniprotKB-P0A077) residue 25-325 were subcloned into popinE vectors (OPPF-UK) resulting in HlgA and HlgB bearing a C-terminal (His) ${ }_{6}$-tag.

Expression and purification of ACKR1. Expression of ACKR1 was carried out in insect and human cells. ACKR1 constructs were expressed in HEK293 GnTI $^{-}$cells (ATCC) using BacMam baculovirus transduction. HEK cells were grown in suspension in Ex-Cell ${ }^{\circledR} 293$ Serum-Free Medium (Sigma) with $2 \% \mathrm{FBS}$ and were infected at a density of $2 \times 10^{6}$ cells $/ \mathrm{mL}$ using a 1/10 (v/v) baculovirus solution. Culture flasks were shaken for $72 \mathrm{~h}$ at $37^{\circ} \mathrm{C}$ with $5 \% \mathrm{CO}_{2}$ and a solution of $5 \mathrm{mM}$ sodium butyrate was added to the culture flasks $24 \mathrm{~h}$ post-infection. Cells were harvested by centrifugation $(3,000 \mathrm{rpm}) 72 \mathrm{~h}$ postinfection and cell pellets were stored at $-80^{\circ} \mathrm{C}$ until purification. ACKR1 constructs were also expressed in Sf9 insect cells (Life Technologies) using the pFastBac baculovirus system (Thermofischer). Sf9 cells were grown in Ex-Cell ${ }^{\circledR} 420$ Medium (Sigma) and were infected at a density of $4 \times 10^{6}$ cells $/ \mathrm{mL}$ using a $1 / 200$ (v/v) baculovirus solution. Culture flasks were shaken for $48 \mathrm{~h}$ at $28^{\circ} \mathrm{C}$ and cells were harvested by centrifugation $(3,000 \mathrm{rpm})$, and cell pellets were stored at $-80{ }^{\circ} \mathrm{C}$ until purification. Purification was carried out in similar conditions regardless cell expression. After thawing the frozen cell pellets, cells were lysed by osmotic shock adding 1/10 (v/v) lysis buffer consisting of $10 \mathrm{mM}$ Tris (pH 7.5), $1 \mathrm{mM}$ EDTA and containing $2 \mathrm{mg} / \mathrm{mL}$ of iodoacetamide (Sigma) and protease inhibitors (Leupeptin (Euromedex), Benzamidine and PMSF (Sigma)). Lysed cells were centrifuged (16,000 rpm) and the membrane pellets were suspended in a 1/20 (v/v) solubilisation buffer consisting of $50 \mathrm{mM} \mathrm{HEPES} \mathrm{(pH} \mathrm{7.5),} 150 \mathrm{mM} \mathrm{NaCl}$, $0.5 \%$ (w/v) n-dodecyl-D-maltoside (DDM, Anatrace), 0.1\% (w/v) cholesteryl-hemi-succinate (CHS, Sigma), $2 \mathrm{mg} / \mathrm{mL}$ of iodoacetamide and protease inhibitors. Receptors were extracted from the membrane pellets using a glass dounce grinder and the extracted mixture was stirred for $1 \mathrm{~h}$ at $4{ }^{\circ} \mathrm{C}$, then centrifuged 
(16,000 rpm). The supernatant was loaded by gravity flow onto anti-Flag M2 antibody resin. The resin was washed with 10 column volumes (CV) of a DDM wash buffer consisting of $50 \mathrm{mM}$ HEPES (pH 7.5), $150 \mathrm{mM} \mathrm{NaCl}, 0.1 \%$ (w/v) DDM and $0.02 \%$ (w/v) CHS. For native MS experiments, DDM concentration was decreased to reach 2 critical micelle concentration (CMC) using $10 \mathrm{CV}$ of wash buffer 2 made up of $50 \mathrm{mM}$ HEPES (pH 7.5), $150 \mathrm{mM} \mathrm{NaCl}, 0.025 \%$ (w/v) DDM and 0.005\% (w/v) CHS. The bound receptor was eluted in the wash buffer 2 supplemented with $0.4 \mathrm{mg} / \mathrm{mL}$ Flag-peptide. For HDX-MS analysis, detergent was changed from DDM to lauryl maltose neopentyl glycol (LMNG) using LMNG exchange buffer containing $50 \mathrm{mM}$ HEPES (pH 7.5), $150 \mathrm{mM} \mathrm{NaCl}, 0.2 \%$ (w/v) LMNG and 0.01\% (w/v) CHS. The detergent exchange was performed by washing the column with a series of 5 buffers ( $3 \mathrm{CV}$ each) made up of the following ratios (v/v) of LMNG exchange buffer and DDM wash buffer: 1/3, 1/1, 3/1, 9/1, 1/0. Additional $10 \mathrm{CV}$ wash was performed to decrease the detergent concentration to 2CMC LMNG using $50 \mathrm{mM}$ HEPES (pH 7.5), $150 \mathrm{mM} \mathrm{NaCl,} \mathrm{0.02 \%} \mathrm{(w/v)} \mathrm{LMNG} \mathrm{and} \mathrm{0.001 \%} \mathrm{(w/v)} \mathrm{CHS} \mathrm{followed} \mathrm{by}$ the last wash LMNG buffer consisting of $50 \mathrm{mM}$ HEPES (pH 7.5), $150 \mathrm{mM} \mathrm{NaCl}, 0.002 \%$ (w/v) LMNG and $0.0001 \%(\mathrm{w} / \mathrm{v})$ CHS. The bound receptor was eluted in the last wash LMNG buffer supplemented with $0.4 \mathrm{mg} / \mathrm{mL}$ Flag-peptide. The eluted solution of receptors was concentrated to $500 \mu \mathrm{L}$ using $50 \mathrm{kDa}$ spin filters (Millipore) and further purified by size exclusion chromatography on a Superdex 200 Increase 10/300 column (GE Healthcare) in the last wash buffer. For PNGase F (NEB) treatment, $5 \mu \mathrm{L}$ of enzyme at $500000 \mathrm{U} / \mathrm{mL}$ were added to $0.2 \mathrm{mg}$ of ACKR1 and incubated overnight at $4{ }^{\circ} \mathrm{C}$. Receptor was purified by size exclusion chromatography as mentioned previously. Fractions containing monodisperse ACKR1 were collected and directly analysed for native MS or pooled and concentrated for HDX analyses.

Expression and purification of HlgA and HlgB. Toxins were expressed with (His) 6 -tags at their C-termini in competent C43 (DE3) Escherichia coli cells (NEB) for HlgA and in BL21 (DE3) Escherichia coli cells (NEB) for HlgB. Transformed cells were grown at $37^{\circ} \mathrm{C}$ in Terrific broth for HlgA and in LB broth for $\mathrm{HlgB}$ supplemented with $100 \mu \mathrm{g} / \mathrm{mL}$ ampicillin to a density of OD600 $=0.6$. Expression was then induced at $22^{\circ} \mathrm{C}$ by addition of $0.5 \mathrm{mM}$ IPTG. Cells were harvested by centrifugation $(3,000 \mathrm{rpm})$ and cell pellets were stored at $-80^{\circ} \mathrm{C}$ until purification. After thawing the frozen cell pellets, cells were lysed by sonication in a lysis buffer consisting of $20 \mathrm{mM}$ Tris (pH 8), $300 \mathrm{mM} \mathrm{NaCl}, 2 \mathrm{mg} / \mathrm{mL}$ of iodoacetamide (Sigma) and protease inhibitors (Leupeptin (Euromedex), Benzamidine and PMSF (Sigma)). Lysed cells were centrifuged (16,000 rpm) and the supernatant was adjusted to $40 \mathrm{mM}$ imidazole and loaded onto a nickel NTA Agarose resin. The resin was washed with $10 \mathrm{CV}$ of wash buffer 1 consisting of $50 \mathrm{mM}$ 
HEPES (pH 7.5) and $1 \mathrm{M} \mathrm{NaCl}$, and with $10 \mathrm{CV}$ of wash buffer 2 consisting of $50 \mathrm{mM} \mathrm{HEPES} \mathrm{(pH} \mathrm{7.5)}$ and $150 \mathrm{mM} \mathrm{NaCl}$ supplemented with $40 \mathrm{mM}$ imidazole. Bound (His)6-toxins were eluted with wash buffer 2 supplemented with $200 \mathrm{mM}$ imidazole. The eluted solution of toxins was concentrated to $500 \mu \mathrm{L}$ using $30 \mathrm{kDa}$ spin filters (Millipore) and further purified by size exclusion chromatography on a Superdex 200 Increase 10/300 column (GE Healthcare) in 50 mM HEPES (pH 7.5) and $150 \mathrm{mM} \mathrm{NaCl}$. Fractions containing monodisperse toxins were collected and concentrated for native MS and HDX analyses and cell-based assays.

Native mass spectrometry. Prior to MS analysis, proteins were buffer exchanged into $200 \mathrm{mM}$ ammonium acetate buffer pH 7.4 (Sigma), supplemented with 0.02\% DDM for ACKR1 and for toxins: detergent interactions analysis, using Bio-Spin microcentrifuge columns (Bio-Rad Laboratories). Intact MS spectra were recorded on a Synapt G1 or a Synapt G2-Si HDMS instrument (Waters Corporation) modified for high mass analysis and operated in ToF mode. Samples were introduced into the ion source using borosilicate emitters (Thermo Scientific). Optimized instrument parameters for ACKR1 alone or in the presence of the toxins were as follows: capillary voltage $1.4 \mathrm{kV}$, sampling cone voltage $150 \mathrm{~V}$, offset voltage $80 \mathrm{~V}$, transfer collision voltage $25 \mathrm{~V}$, argon flow rate $8 \mathrm{~mL} / \mathrm{min}$ and trap bias $25 \mathrm{~V}$. Collision voltage in the trap was optimized between 50 and $110 \mathrm{~V}$ depending on the sample, and to the minimum activation required to strip the detergent micelle when it was present. Membrane proteins are ionized bound to the detergent micelle and therefore require activation by collision-induced dissociation in order to dissociate bound detergents (35). Data was processed using MassLynx v.4.2 (Waters) and UniDec (64).

Transfection for cell-based assays. HEK293 cells (ATCC) were grown in Dulbecco's Modified Eagle Medium supplemented with $10 \% \mathrm{FBS}$ and $1 \%$ Penicillin/streptomycin at $37^{\circ} \mathrm{C}$ with $5 \% \mathrm{CO}_{2}$. Transfection was performed using lipofectamine 2000 (Life technology) in polyornithine coated black-walled, darkbottom 96-well plates. The quantity of transfected DNA was optimised for each construct and $150 \mathrm{ng}$ of DNA in total, supplemented by empty PRK5, were added to $50.10^{4}$ cells/well for transfection.

Competitive binding assay by TR-FRET. $1.5 \mathrm{ng}$ of SNAP-ACKR1 were added to $50.10^{4}$ cells/well for transfection. 48 h post-transfection, the plate was washed twice with TagLite ${ }^{\circledR}$ (Cisbio, Codolet, France) and the extracellular SNAP-receptors were labelled with SNAP-Lumi4-Tb (100 nM, Cisbio, Codolet, France) for $1 \mathrm{~h}$ at $37^{\circ} \mathrm{C}$. The plate was washed three times with TagLite ${ }^{\circledR} .12 \mathrm{nM}$ of d2-labelled CCL5 and increasing concentration $(0,1,3.6,10,31.6,100,316,1000,3160$ and $10000 \mathrm{nM})$ of toxins, HlgA, HlgB 
or an equimolar mixture of $\mathrm{HlgA}$ and $\mathrm{HlgB}$, were added to cells. Lumi-4-fluorescence signals were observed on a Pherastar plate reader (BMG Labtech): samples were illuminated at $337 \mathrm{~nm}$ and fluorescence was acquired at $620 \mathrm{~nm}$ (donor) and $665 \mathrm{~nm}$ (TR-FRET) over time. The ratio of the signals $(665 / 620)$ was plotted versus the toxin concentration. Dose-response curves were generated using GraphPad Prism $6{ }^{\circledR}$ (GraphPad Software, Inc., San Diego, CA).

Receptor-receptor interactions by BRET. $80 \mathrm{ng}$ of ACKR1-YFP and $10 \mathrm{ng}$ of ACKR1-NanoLuc were added to $50.10^{4}$ cells/well for transfection. $24 \mathrm{~h}$ post-transfection, the plate was washed three times with Phosphate Buffered Saline (PBS, Sigma) and then cells are pre-incubated 5 minutes at $37^{\circ} \mathrm{C}$ with $5 \mu \mathrm{M}$ coelenterazine $\mathrm{h}$ (Promega) before adding ligands diluted in PBS supplemented with $0.9 \mathrm{mM} \mathrm{CaCl} 2$ and $0.5 \mathrm{mM} \mathrm{MgCl}_{2}$. Increasing concentration $(0,1,3.6,10,31.6,100,316,1000,3160$ and $10000 \mathrm{nM})$ of toxins, HlgA, HlgB or an equimolar mixture of $\mathrm{HlgA}$ and $\mathrm{HlgB}$, were added to cells. BRET readings were collected using a Mithras 2 plate reader (Berthold Technologies GmbH, Bad Wildbad, Germany) with 0.1 seconds integration time per well. The reading chamber was maintained at $37^{\circ} \mathrm{C}$ throughout the entire reading time. The BRET signal was calculated by the ratio of emission of YFP (535nm) to NanoLuc (460nm). Dose-response curves were generated using GraphPad Prism $6^{\circledR}$ (GraphPad Software, Inc., San Diego, CA).

Receptor-receptor interactions by TR-FRET. $50 \mathrm{ng}$ of SNAP-ACKR1 or SNAP-ACKR3 (control) were added to $50.10^{4}$ cells/well for transfection. $24 \mathrm{~h}$ post-transfection, the plate was washed twice with TagLite $^{\circledR}$ (Cisbio, Codolet, France) and the extracellular SNAP-receptors were labelled with SNAPLumi4-Tb (100 nM, Cisbio, Codolet, France) and SNAP-red (300 nM, Cisbio, Codolet, France) for $1 \mathrm{~h}$ at $37^{\circ} \mathrm{C}$. The plate was washed four times with PBS. Increasing concentration $(0,1,3.6,10,31.6,100,316$, 1000, 3160 and $10000 \mathrm{nM}$ ) of toxins, HlgA, HlgB or an equimolar mixture of $\mathrm{HlgA}$ and $\mathrm{HlgB}$, were then added to cells. 30 minutes after addition of leukotoxins, TRF readings were collected using a SPARK $20 \mathrm{M}$ plate reader (TECAN) with $150 \mu \mathrm{s}$ of lag time and $500 \mu \mathrm{s}$ of integration time per well. Excitation at $340 \mathrm{~nm}$ and emission at $620 \mathrm{~nm}$ (donor) and $665 \mathrm{~nm}$ (TR-FRET) over time. The ratio of the signals $(665 / 620)$ was plotted versus the concentrations of toxin.

Receptor-Ga interactions by BRET. To follow interaction between ACKR1 and Ga subunit, $40 \mathrm{ng}$ of ACKR1-YFP and $10 \mathrm{ng}$ of Ga-RLuc were added to $50.10^{4}$ cells/well for transfection. To follow G protein activation, cells were transfected with each ST-Receptor (10 ng CCR5, $10 \mathrm{ng}$ CCR2, $20 \mathrm{ng}$ ACKR1) and 
in parallel with $10 \mathrm{ng}$ Gai1-RLuc, $10 \mathrm{ng} \beta 2$ and $20 \mathrm{ng} \gamma$-Venus. $24 \mathrm{~h}$ post-transfection, the plate was washed three times with Phosphate Buffered Saline (PBS, Sigma) and then cells were pre-incubated 5 minutes at $37^{\circ} \mathrm{C}$ with $5 \mu \mathrm{M}$ coelenterazine $\mathrm{h}$ (Promega) before adding ligands diluted in PBS supplemented with $0.9 \mathrm{mM} \mathrm{CaCl}_{2}$ and $0.5 \mathrm{mM} \mathrm{MgCl}_{2}$. BRET readings were collected using a Mithras 2 plate reader (Berthold Technologies $\mathrm{GmbH}$, Bad Wildbad, Germany) with 0.1 seconds integration time per well. The reading chamber was maintained at $37^{\circ} \mathrm{C}$ throughout the entire reading time. The BRET signal was calculated by the ratio of emission of YFP $(535 \mathrm{~nm})$ to RLuc $(480 \mathrm{~nm})$. Dose-response curves were generated using GraphPad Prism $6^{\circledR}$ (GraphPad Software, Inc., San Diego, CA).

Hydrogen-Deuterium exchange mass spectrometry. HDX-MS experiments were performed using a Synapt G2-Si HDMS coupled to nanoAQUITY UPLC with HDX Automation technology (Waters Corporation). ACKR1 in LMNG detergent was concentrated up to 10-20 $\mu \mathrm{M}$ and optimization of the sequence coverage was performed on undeuterated controls. Various quench times and conditions were tested, i.e. in the presence or absence of different denaturing or reducing reagents with or without longer trapping times in order to wash them out. The best sequence coverage and redundancy for ACKR1 were systematically obtained without the addition of any denaturing or reducing agents in the quench buffer. Mixtures of receptor: leukotoxins were pre-incubated together to reach equilibrium prior to HDX-MS analysis. Analysis of freshly prepared ACKR1 apo, ACKR1: HlgA and ACKR1: HlgB (1: 1 ratio) mixtures were performed as follows: $3 \mu \mathrm{L}$ of sample are diluted in $57 \mu \mathrm{L}$ of undeuterated for the reference or deuterated last wash SEC buffer. The final percentage of deuterium in the deuterated buffer was $95 \%$. Deuteration was performed at $20^{\circ} \mathrm{C}$ for $30,60,300,600$ and $1800 \mathrm{sec}$. Next, $50 \mu \mathrm{L}$ of reaction sample are quenched in $50 \mu \mathrm{L}$ of quench buffer $\left(\mathrm{KH}_{2} \mathrm{PO}_{4} 50 \mathrm{mM}\right.$ and $\mathrm{K}_{2} \mathrm{HPO}_{4} 50 \mathrm{mM}$, pH 2.3) at $0^{\circ} \mathrm{C} .80 \mu \mathrm{L}$ of quenched sample are loaded onto a $50 \mu \mathrm{L}$ loop and injected on an Enzymate pepsin column $(300 \AA$, $5 \mu \mathrm{m}$, $2.1 \mathrm{~mm}$ X $30 \mathrm{~mm}$, Waters) maintained at $15^{\circ} \mathrm{C}$, with $0.2 \%$ formic acid at a flowrate of $100 \mu \mathrm{L} / \mathrm{min}$ and an additional backing pressure of 6000 psi controlled by the HDX regulator kit (Waters). The peptides are then trapped at $0^{\circ} \mathrm{C}$ on a Vanguard column (ACQUITY UPLC BEH C18 VanGuard Pre-column, 130A, $1.7 \mu \mathrm{m}, 2.1 \mathrm{~mm}$ X $5 \mathrm{~mm}$, Waters) for $3 \mathrm{~min}$, before being loaded at $40 \mu \mathrm{L} / \mathrm{min}$ onto an Acquity UPLC column (ACQUITY UPLC BEH C18 Column, $1.7 \mu \mathrm{m}, 1 \mathrm{~mm}$ X $100 \mathrm{~mm}$, Waters) kept at $0^{\circ} \mathrm{C}$. Peptides are subsequently eluted with a linear gradient $(0.2 \%$ formic acid in acetonitrile solvent at $5 \%$ up to $35 \%$ during the first $6 \mathrm{~min}$, then up to $40 \%$ and $95 \%$ over $1 \mathrm{~min}$ each) and ionized directly by electrospray on a Synapt G2-Si mass spectrometer (Waters). HDMS ${ }^{\mathrm{E}}$ data were obtained by 20-30 V trap collision energy 
ramp. Lock mass accuracy correction was made using a mixture of leucine enkephalin. The pepsin column was then washed three times with Guanidine- $\mathrm{HCl} 1.5 \mathrm{M}$, acetonitrile $4 \%$ and formic acid $0.8 \%$ and a blank is performed between each sample in order to minimize the carry-over. All timepoints were performed in triplicates.

Peptide identification was performed from undeuterated data using ProteinLynx global Server (PLGS, version 3.0.3, Waters). Peptides are filtered by DynamX (version 3.0, Waters) using the following parameters: minimum intensity of 1000 , minimum product per amino acid of 0.2 , maximum error for threshold of $5 \mathrm{ppm}$. All peptides were manually checked and data was curated using DynamX. Maximally labelled control was not performed and back exchange was not corrected since we are measuring differential HDX and not absolute one (44). Some peptides, mainly present in unstructured and flexible regions, presented an additional long-exposure back exchange since the LEAP robot skips a cleaning step in non-deuterated buffer for deuteration timepoints shorter than 2 min (65). Statistical analysis of all $\triangle$ HDX data was performed using Deuteros 2.0 (66) and only peptides with a 99\% confidence interval were considered. 


\section{Acknowledgments and funding sources}

The authors would like to thank Isabel Brabet for her help with the cell-based assays and Dr. Kallol Gupta for critical reading of the manuscript. CB would like to thank James Sturgis and all the members of the "Plateforme Protéomique" at the IMM in Marseille for giving her access to the Synapt for preliminary data acquisitions. Mass spectrometry experiments were carried out using the facilities of the Montpellier Proteomics Platform (PPM, BioCampus Montpellier) and the Plateforme Protéomique (IMM, Marseille). All cell-based assays were carried out at the Arpège facility (BioCampus, Montpellier). This work was supported by the regional funds FEDER/Région Occitanie, MUSE, Labex EpiGenMed and the French Agence Nationale de la Recherche (project ANR-17-CE15-0002-01, CHEMSPEC). 


\section{References}

1. N. A. Turner, et al., Methicillin-resistant Staphylococcus aureus: an overview of basic and clinical research. Nat Rev Microbiol 17, 203-218 (2019).

2. T. J. Foster, The Staphylococcus aureus “superbug." Journal of Clinical Investigation 114, 1693-1696 (2004).

3. L. M. Assis, M. Nedeljković, A. Dessen, New strategies for targeting and treatment of multi-drug resistant Staphylococcus aureus. Drug Resistance Updates 31, 1-14 (2017).

4. C. Kong, H. Neoh, S. Nathan, Targeting Staphylococcus aureus Toxins: A Potential form of Anti-Virulence Therapy. Toxins 8, 72 (2016).

5. E. S. Seilie, J. Bubeck Wardenburg, Staphylococcus aureus pore-forming toxins: The interface of pathogen and host complexity. Seminars in Cell \& Developmental Biology 72, 101-116 (2017).

6. F. C. O. Los, T. M. Randis, R. V. Aroian, A. J. Ratner, Role of Pore-Forming Toxins in Bacterial Infectious Diseases. Microbiology and Molecular Biology Reviews 77, 173-207 (2013).

7. F. Alonzo, V. J. Torres, The Bicomponent Pore-Forming Leucocidins of Staphylococcus aureus. Microbiology and Molecular Biology Reviews 78, 199-230 (2014).

8. F. Vandenesch, G. Lina, T. Henry, Staphylococcus aureus Hemolysins, bi-component Leukocidins, and Cytolytic Peptides: A Redundant Arsenal of Membrane-Damaging Virulence Factors? Frontiers in Cellular and Infection Microbiology 2 (2012).

9. K. Yamashita, et al., Crystal structure of the octameric pore of staphylococcal -hemolysin reveals the -barrel pore formation mechanism by two components. Proceedings of the National Academy of Sciences 108, 17314-17319 (2011).

10. D. Yamashita, et al., Molecular basis of transmembrane beta-barrel formation of staphylococcal pore-forming toxins. Nature Communications 5 (2014).

11. J. Kaneko, Y. Kamio, Bacterial Two-component and Hetero-heptameric Pore-forming Cytolytic Toxins: Structures, Pore-forming Mechanism, and Organization of the Genes. Bioscience, Biotechnology, and Biochemistry 68, 981-1003 (2004).

12. A. Lubkin, et al., Staphylococcus aureus Leukocidins Target Endothelial DARC to Cause Lethality in Mice. Cell Host \& Microbe 25, 463-470.e9 (2019).

13. A. N. Spaan, et al., Staphylococcus aureus Targets the Duffy Antigen Receptor for Chemokines (DARC) to Lyse Erythrocytes. Cell Host \& Microbe 18, 363-370 (2015).

14. A. N. Spaan, et al., The staphylococcal toxins $\gamma$-haemolysin AB and CB differentially target phagocytes by employing specific chemokine receptors. Nature Communications 5 (2014).

15. T. Reyes-Robles, et al., Staphylococcus aureus Leukotoxin ED Targets the Chemokine Receptors CXCR1 and CXCR2 to Kill Leukocytes and Promote Infection. Cell Host \& Microbe 14, 453-459 (2013).

16. F. Alonzo III, et al., CCR5 is a receptor for Staphylococcus aureus leukotoxin ED. Nature 493, 51-55 (2012).

17. A. N. Spaan, J. A. G. van Strijp, V. J. Torres, Leukocidins: staphylococcal bi-component pore-forming toxins find their receptors. Nature Reviews Microbiology 15, 435-447 (2017). 
18. T. Ozawa, J. Kaneko, Y. Kamio, Essential Binding of LukF of Staphylococcal $\gamma$-Hemolysin Followed by the Binding of $\mathrm{H} \gamma$ II for the Hemolysis of Human Erythrocytes. Bioscience, Biotechnology, and Biochemistry 59, 1181-1183 (1995).

19. P. Yoong, V. J. Torres, Counter inhibition between leukotoxins attenuates Staphylococcus aureus virulence. Nature Communications 6 (2015).

20. A. T. Tromp, et al., Human CD45 is an F-component-specific receptor for the staphylococcal toxin Panton-Valentine leukocidin. Nature Microbiology 3, 708-717 (2018).

21. A. T. Tromp, J. A. G. van Strijp, Studying Staphylococcal Leukocidins: A Challenging Endeavor. Frontiers in Microbiology 11 (2020).

22. R. Horuk, The Duffy Antigen Receptor for Chemokines DARC/ACKR1. Frontiers in Immunology 6 (2015).

23. R. J. B. Nibbs, G. J. Graham, Immune regulation by atypical chemokine receptors. Nature Reviews Immunology 13, 815-829 (2013).

24. I. Novitzky-Basso, A. Rot, Duffy antigen receptor for chemokines and its involvement in patterning and control of inflammatory chemokines. Front. Immun. 3 (2012).

25. C. Cancellieri, A. Vacchini, M. Locati, R. Bonecchi, E. M. Borroni, Atypical chemokine receptors: from silence to sound. Biochemical Society Transactions 41, 231-236 (2013).

26. C. A. H. Hansell, C. E. Hurson, R. J. B. Nibbs, DARC and D6: silent partners in chemokine regulation? Immunol Cell Biol 89, 197-206 (2011).

27. M. Pruenster, et al., The Duffy antigen receptor for chemokines transports chemokines and supports their promigratory activity. Nat Immunol 10, 101-108 (2009).

28. A. Vacchini, M. Locati, E. M. Borroni, Overview and potential unifying themes of the atypical chemokine receptor family. Journal of Leukocyte Biology 99, 883-892 (2016).

29. I. Kufareva, M. Gustavsson, Y. Zheng, B. S. Stephens, T. M. Handel, What Do Structures Tell Us About Chemokine Receptor Function and Antagonism? Annual Review of Biophysics 46, 175-198 (2017).

30. M. Arimont, et al., Structural Analysis of Chemokine Receptor-Ligand Interactions. Journal of Medicinal Chemistry 60, 4735-4779 (2017).

31. Z. Peng, et al., Rim domain loops of staphylococcal $\beta$-pore forming bi-component toxin S-components recognize target human erythrocytes in a coordinated manner. The Journal of Biochemistry 164, 93-102 (2018).

32. M. T. Vasquez, et al., Identification of a domain critical for Staphylococcus aureus LukED receptor targeting and lysis of erythrocytes. Journal of Biological Chemistry 295, 17241-17250 (2020).

33. H. Nariya, Y. Kamio, Identification of the Minimum Segment Essential for the $\mathrm{H} \gamma$ ll-Specific Function of Staphylococcal $\gamma$-Hemolysin. Bioscience, Biotechnology, and Biochemistry 61, 1786-1788 (1997).

34. T. M. Allison, C. Bechara, Structural mass spectrometry comes of age: new insight into protein structure, function and interactions. Biochemical Society Transactions 47, 317-327 (2019).

35. C. Bechara, C. V. Robinson, Different Modes of Lipid Binding to Membrane Proteins Probed by Mass Spectrometry. Journal of the American Chemical Society 137, 5240-5247 (2015). 
36. C. Martens, A. Politis, A glimpse into the molecular mechanism of integral membrane proteins through hydrogendeuterium exchange mass spectrometry. Protein Science (2020) https:/doi.org/10.1002/pro.3853 (May 12, 2020).

37. J. R. Engen, T. Botzanowski, D. Peterle, F. Georgescauld, T. E. Wales, Developments in Hydrogen/Deuterium Exchange Mass Spectrometry. Analytical Chemistry 93, 567-582 (2020).

38. A. N. Calabrese, S. E. Radford, Mass spectrometry-enabled structural biology of membrane proteins. Methods 147, 187-205 (2018).

39. J. E. Keener, G. Zhang, M. T. Marty, Native Mass Spectrometry of Membrane Proteins. Anal. Chem., acs.analchem.0c04342 (2020).

40. H.-Y. Yen, et al., Ligand binding to a G protein-coupled receptor captured in a mass spectrometer. Science Advances 3, e1701016 (2017).

41. H.-Y. Yen, et al., PtdIns(4,5)P2 stabilizes active states of GPCRs and enhances selectivity of G-protein coupling. Nature 559, 423-427 (2018).

42. J. M. Zwier, et al., A Fluorescent Ligand-Binding Alternative Using Tag-lite ${ }^{\circledR}$ Technology. J Biomol Screen 15, 12481259 (2010).

43. G. Vauquelin, S. J. Charlton, Exploring avidity: understanding the potential gains in functional affinity and target residence time of bivalent and heterobivalent ligands: Exploring bivalent ligand binding properties. Br J Pharmacol 168, 1771-1785 (2013).

44. G. R. Masson, et al., Recommendations for performing, interpreting and reporting hydrogen deuterium exchange mass spectrometry (HDX-MS) experiments. Nature Methods 16, 595-602 (2019).

45. M. Chabre, M. le Maire, Monomeric G-Protein-Coupled Receptor as a Functional Unit. Biochemistry 44, 9395-9403 (2005).

46. M. R. Whorton, et al., A monomeric G protein-coupled receptor isolated in a high-density lipoprotein particle efficiently activates its G protein. PNAS 104, 7682-7687 (2007).

47. J. Zheng, T. Strutzenberg, B. D. Pascal, P. R. Griffin, Protein dynamics and conformational changes explored by hydrogen/deuterium exchange mass spectrometry. Current Opinion in Structural Biology 58, 305-313 (2019).

48. Y. Du, et al., Assembly of a GPCR-G Protein Complex. Cell 177, 1232-1242.e11 (2019).

49. F. Fiorentino, et al., Dynamics of an LPS translocon induced by substrate and an antimicrobial peptide. Nature Chemical Biology 17, 187-195 (2020).

50. R. Jia, et al., Hydrogen-deuterium exchange mass spectrometry captures distinct dynamics upon substrate and inhibitor binding to a transporter. Nature Communications 11 (2020).

51. I. R. Möller, et al., Conformational dynamics of the human serotonin transporter during substrate and drug binding. Nature Communications 10 (2019).

52. E. Reading, et al., Perturbed structural dynamics underlie inhibition and altered efflux of the multidrug resistance pump AcrB. Nature Communications 11 (2020).

53. X. Zhang, et al., Dynamics of the $\beta_{2}$-Adrenergic G-Protein Coupled Receptor Revealed by Hydrogen-Deuterium Exchange. Analytical Chemistry 82, 1100-1108 (2010).

54. N. R. Latorraca, A. J. Venkatakrishnan, R. O. Dror, GPCR Dynamics: Structures in Motion. Chem. Rev., 17 (2017). 
55. J. P. Mahoney, R. K. Sunahara, Mechanistic insights into GPCR-G protein interactions. Curr Opin Struct Biol 41, 247254 (2016).

56. H. Zhang, et al., Structural basis for selectivity and diversity in angiotensin II receptors. Nature 544, 327-332 (2017).

57. P. M. Dijkman, et al., Conformational dynamics of a G protein-coupled receptor helix 8 in lipid membranes. Science Advances 6, eaav8207 (2020).

58. L. Albizu, et al., Time-resolved FRET between GPCR ligands reveals oligomers in native tissues. Nat Chem Biol 6, 587-594 (2010).

59. N. E. Brown, J. B. Blumer, J. R. Hepler, "Bioluminescence Resonance Energy Transfer to Detect Protein-Protein Interactions in Live Cells" in Protein-Protein Interactions, Methods in Molecular Biology., C. L. Meyerkord, H. Fu, Eds. (Springer New York, 2015), pp. 457-465.

60. D. Maurel, et al., Cell-surface protein-protein interaction analysis with time-resolved FRET and snap-tag technologies: application to GPCR oligomerization. Nature Methods 5, 561-567 (2008).

61. P. R. Selvin, Principles and biophysical applications of lanthanide-based probes. Annu Rev Biophys Biomol Struct 31, 275-302 (2002).

62. T.-H. Lan, et al., BRET evidence that $\beta 2$ adrenergic receptors do not oligomerize in cells. Sci Rep 5, 10166 (2015).

63. A. Chakera, R. M. Seeber, A. E. John, K. A. Eidne, D. R. Greaves, The Duffy Antigen/Receptor for Chemokines Exists in an Oligomeric Form in Living Cells and Functionally Antagonizes CCR5 Signaling through HeteroOligomerization. Molecular Pharmacology 73, 1362-1370 (2008).

64. M. T. Marty, et al., Bayesian Deconvolution of Mass and Ion Mobility Spectra: From Binary Interactions to Polydisperse Ensembles. Anal. Chem. 87, 4370-4376 (2015).

65. R. J. Lumpkin, E. A. Komives, DECA, A Comprehensive, Automatic Post-processing Program for HDX-MS Data*. Molecular \& Cellular Proteomics 18, 2516-2523 (2019).

66. A. M. Lau, J. Claesen, K. Hansen, A. Politis, Deuteros 2.0: peptide-level significance testing of data from hydrogen deuterium exchange mass spectrometry. Bioinformatics, btaa677 (2020).

67. P. Lössl, J. Snijder, A. J. R. Heck, Boundaries of Mass Resolution in Native Mass Spectrometry. J. Am. Soc. Mass Spectrom. 25, 906-917 (2014). 


\section{Figure legends}

Figure 1. Native MS spectra of leukotoxins and ACKR1. A) nMS spectrum of $30 \mu \mathrm{M}$ HlgA and B) 15 $\mu \mathrm{M}$ HlgB showing the presence of both monomeric (33 $004 \pm 1$ Da and $34943 \pm 1$ Da respectively) and dimeric (66 $061 \pm 14$ Da and $69972 \pm 10$ Da respectively) species. Several sodium adducts were visible with both toxins, and could be resolved for the monomeric but not for the dimeric peaks due to the decay in the apparent resolution when working in nMS mode (67). Theoretical masses of HlgA monomer and dimer: 33004 and 66008 Da. Theoretical masses of HlgB monomer and dimer are 34943 and 69886 Da. Single and double circles showed at similar $\mathrm{m} / \mathrm{z}$ regions correspond to overlapping signals coming from monomers and homodimers. C) nMS spectrum of a mixture of $10 \mu \mathrm{M} H \operatorname{HgA}$ and $10 \mu \mathrm{M}$ HlgB showing the additional presence of $\mathrm{HlgAB}$ heterodimers (69 $925 \pm 9 \mathrm{Da}$ ). Overlapping signal from $\mathrm{HlgB} 9+$, HlgBB $18+$ and $\mathrm{HlgAA} 17+$ is visible at $\mathrm{m} / \mathrm{z}$ around 3885 . Bar diagram shows the relative quantification in this equimolar mixture, relative to the most intense species in the spectrum (i.e. HlgA monomer). D) SEC profile of purified ACKR1. E) nMS spectrum of monomeric and F) dimeric ACKR1 produced in HEK GnTI $^{-}$cells (40 $531 \pm 32 \mathrm{Da}$ and $81032 \pm 50 \mathrm{Da}$ respectively) showing an additional $\sim 3.5 \mathrm{kDa}$ glycosylations per monomer. All samples were buffer exchanged in $200 \mathrm{mM}$ ammonium acetate $\mathrm{pH} 7.4$ supplemented with $2 \mathrm{CMC}$ DDM prior to nMS analysis. Single green circles HlgA, double green circles HlgAA, single yellow circles $\mathrm{HlgB}$, double yellow circles $\mathrm{HlgBB}$, double green and yellow circles $\mathrm{HlgAB}$, single blue circles ACKR1 WT, double blue circles ACKR1 homodimer.

Figure 2. Binding of leukotoxins to ACKR1 in vitro and in living cells. A) nMS spectrum of a mixture of $2 \mu \mathrm{M}$ HlgA and $5 \mu \mathrm{M}$ ACKR1 treated with PNGase, showing the presence of monomeric HlgA (green circles, $33004 \pm 1 \mathrm{Da}$ ), deglycosylated ACKR1 (blue circles, $37022 \pm 1 \mathrm{Da}$ ) and partially-hydrolysed deglycosylated ACKR1 (light blue circles, $36411 \pm 1 \mathrm{Da}$ ). Complexes formed between HlgA and both forms of deglycosylated ACKR1 are labelled with dark and clear red stars (70 029 \pm 2 Da and $69421 \pm 7$ Da respectively). B) Scheme explaining TR-FRET competitive binding assay (top) and dose-response curves showing the decrease in TR-FRET ratio between ACKR1 and d2-CCL5 upon addition of HlgA, $\mathrm{HlgB}$ and $\mathrm{HlgAB}$ equimolar mixture (bottom). Data shown are the mean +/- SEM of one experiment performed in triplicates and are representative of 3 independent experiments. Hill slope values: HlgA $1.1 \pm 0.08$, HlgB $-2.0 \pm 0.34$, HlgAB -2.1 \pm 0.29 . IC50 values: HlgA $577 \pm 93$ nM, HlgB $376 \pm 105$ nM, $\mathrm{HlgAB} 6.2 \pm 2.7 \mathrm{nM}$. Values represents the average $+/-\mathrm{SD}$ of three independent experiments performed in triplicates.

Figure 3. ACKR1 conformational changes upon binding to leukotoxins probed by HDX. HDX results showing statistically significant $\triangle \mathrm{HDX}$ regions from all biological replicates, colour-coded onto the snakeplot of ACKR1 adapted from GPCRdb as well as on the structural model generated for ACKR1 where the long $\mathrm{N}$-terminal domain is missing (c.f. methods). Blue: protected regions, grey: regions with no statistically significant $\triangle \mathrm{HDX}$ and white: regions with no HDX data. Deuterium uptake of selected peptides is shown for the apo receptor (black) and the receptor bound to HlgA (green) or to HlgB (yellow). Uptake plot data are the average and standard deviation for timepoints from $n=3$ replicate measurments for one biological preparation of ACKR1.

Figure 4. HIgB and HIgAB interfere with preassembled ACKR1-Gai1 complexes in living cells. A) Scheme explaining BRET assay used to probe ACKR1-Ga interactions. B) Net BRET assay between ACKR1-YFP and Gai1-RLuc showing that CCL5 and HlgA has no specific effect on ACKR1-Gai1 interactions at the tested concentrations, whereas adding $\mathrm{HlgB}$ or $\mathrm{Hlg} \mathrm{AB}$ resulted in the dissociation of 
ACKR1-Gai1 complexes in living cells. EC50 was $796 \pm 110 \mathrm{nM}$ for $\mathrm{HlgB}$ and $13.5 \pm 3.2 \mathrm{nM}$ for HlgAB. C) Net BRET assay showing the effect of HlgAB mixture on ACKR1-Gail interactions for WT, Y30F and Y41F ACKR1. Effect of HIgAB on the dissociation between ACKR1 and Gail decreased when mutanting Y41, evidenced by an EC50 increase up to $106 \pm 42 \mathrm{nM}$, whereas the EC50 did not change significantly when mutating Y30 $(21.7 \pm 5.3 \mathrm{nM})$. Cell-based assays data shown are the mean +/- SEM of one experiment performed in triplicates and are representative of 3 independent experiments. See supplementary Fig. S7 for additional data.

Figure 5. Effect of leukotoxins on ACKR1-ACKR1 interactions in living cells. A) BRET assay showing receptor-receptor interactions at the C-terminal intracellular side of ACKR1. Here also, a constitutive BRET signal is visible prior to adding the different ligands. Adding HlgA (green) does not affect the BRET signal at the tested conditions, whereas $\mathrm{HlgB}$ (yellow) and equimolar mixture of $\mathrm{HlgAB}$ (orange) induced an increased BRET. Average EC50 of all independent experiments was: $178 \pm 28 \mathrm{nM}$ in the presence of $\mathrm{HlgB}$ and $18.6 \pm 2.5 \mathrm{nM}$ in the presence of HlgAB. B) TR-FRET showing receptor-receptor interactions at the $\mathrm{N}$-terminal extracellular side of ACKR1. A constitutive TR-FRET signal visible prior to adding the different ligands. Adding HlgA (green) does not affect the TR-FRET at the tested conditions, whereas $\mathrm{HlgB}$ (yellow) and equimolar mixture of $\mathrm{Hlg} \mathrm{AB}$ (orange) induced a decreased TR-FRET. Average EC50 of all independent experiments was: $429 \pm 138 \mathrm{nM}$ in the presence of $\mathrm{HlgB}$ and $47 \pm 15 \mathrm{nM}$ in the presence of HlgAB. ST, SNAP Tag, ACKR3 was used as control. All data shown are the mean $+/$ - SEM of one experiment performed in triplicates and are representative of at least 3 independent experiments.

Figure 6. Proposed first steps of pore-formation by HlgAB. ACKR1 (blue) is present in both monomeric and dimeric forms in cellular membranes. Soluble HlgA (green) and $\mathrm{HlgB}$ (olive) secreted by SA can be present in monomeric and dimeric forms. Both leukotoxins recognise the cellular membrane by specific interactions with ACKR1, and interaction of each toxin with ACKR1 will lead to conformational changes at both $\mathrm{N}$ - and $\mathrm{C}$-termini of the GPCR. HlgB homodimers and $\mathrm{HIgAB}$ heterodimers could interfere with receptor-receptor interactions, but only HlgAB-(ACKR1) 2 complex will lead to the formation of the pore. 

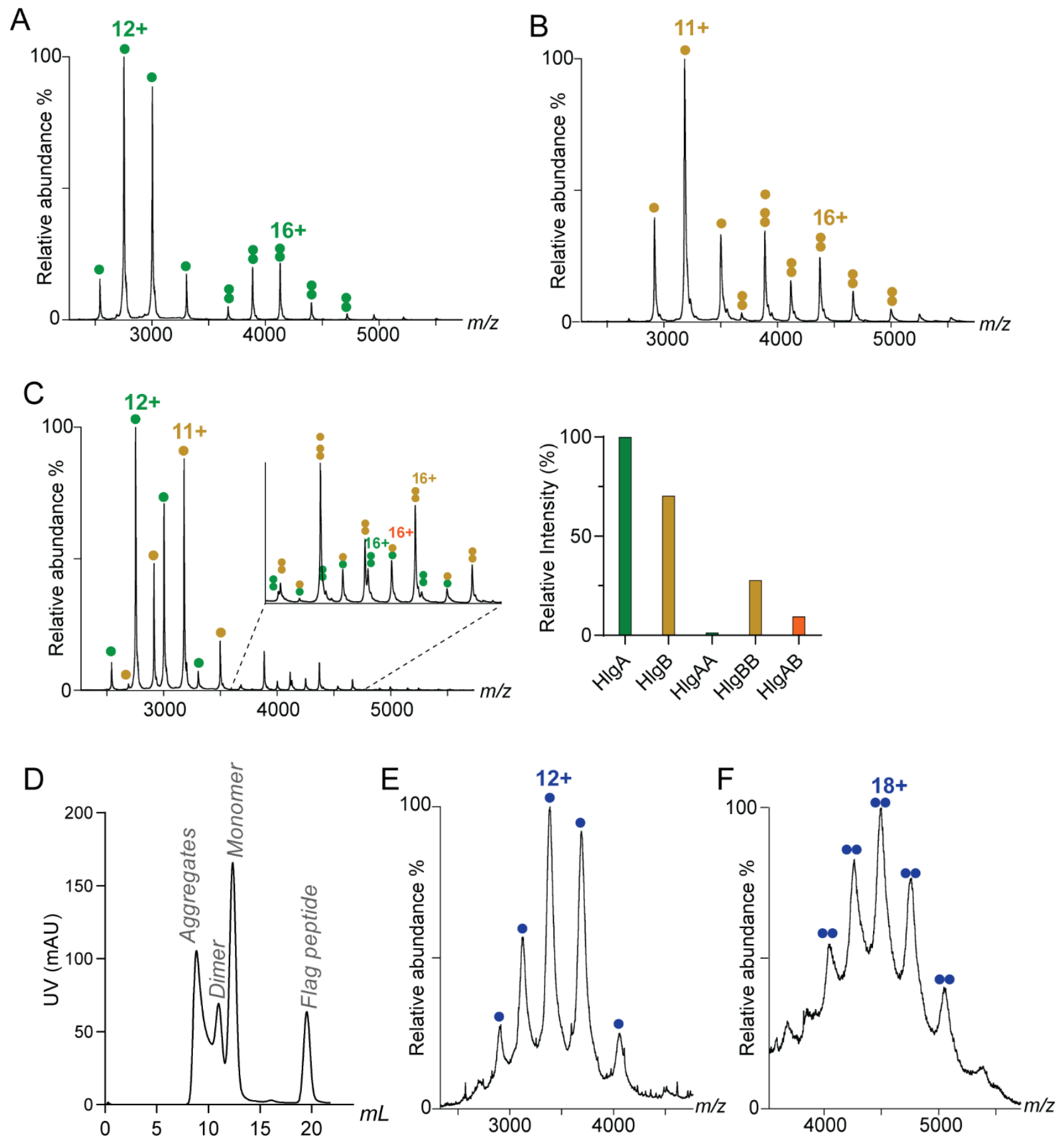

Figure 1 

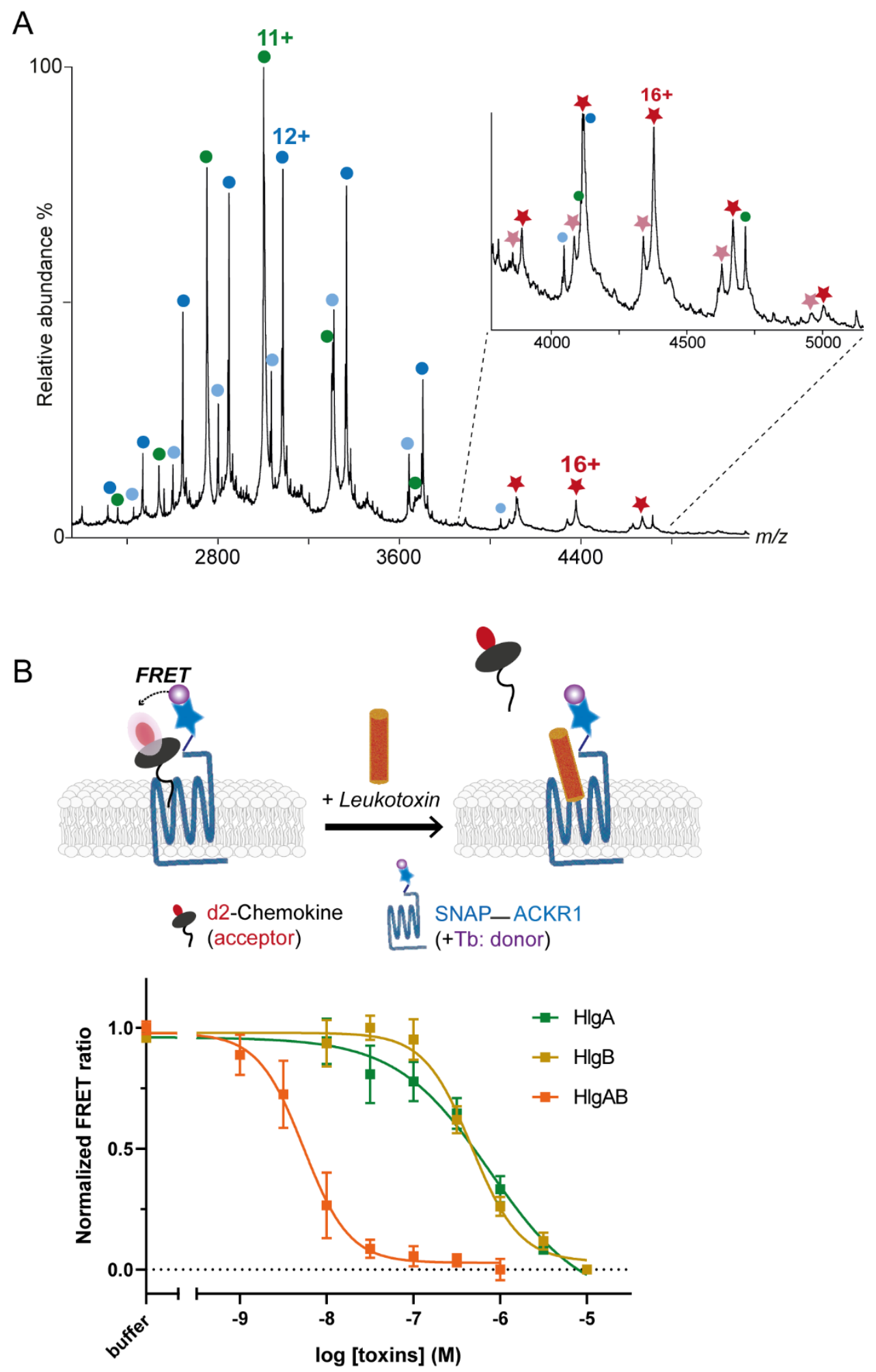

Figure 2 


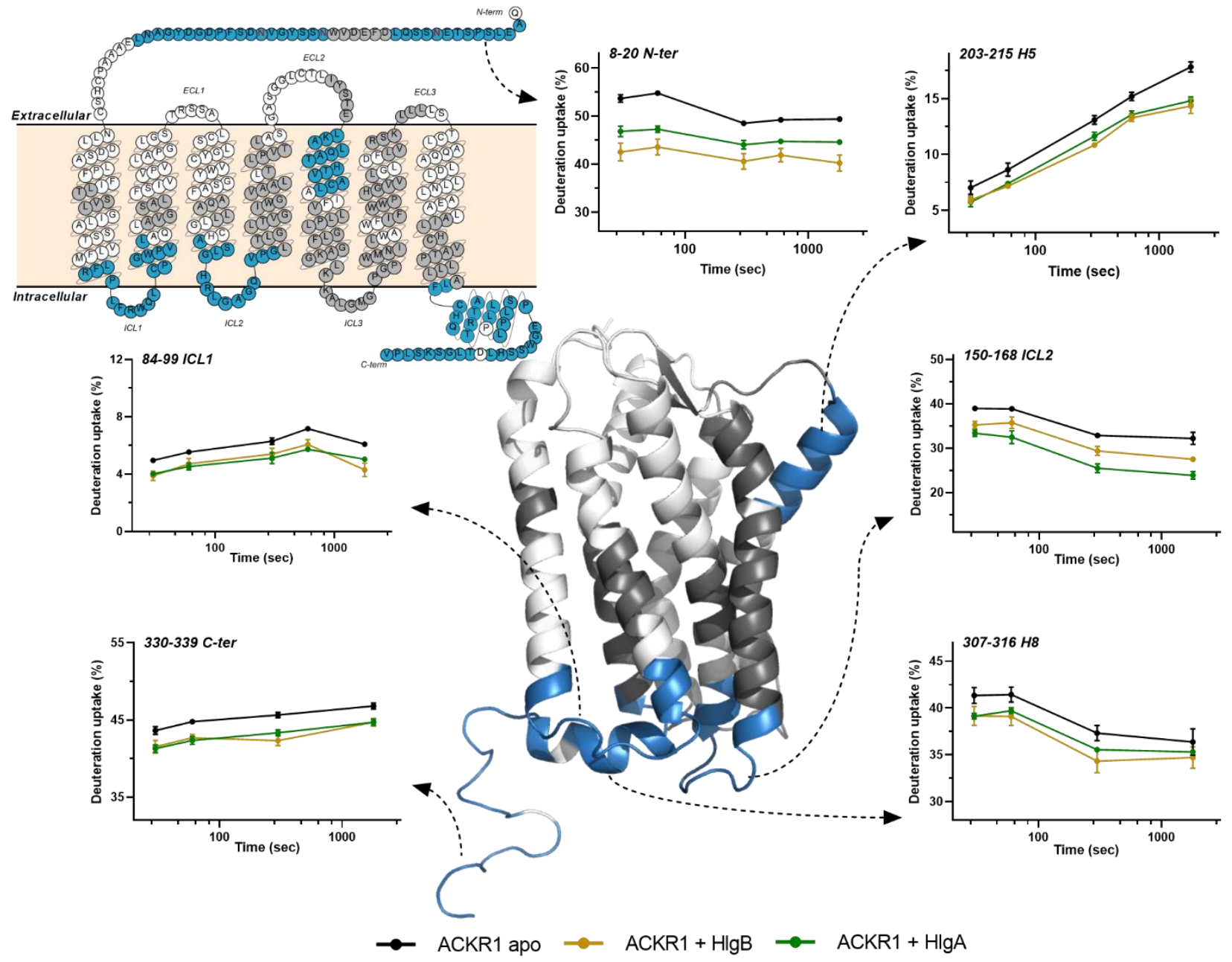

Figure 3 


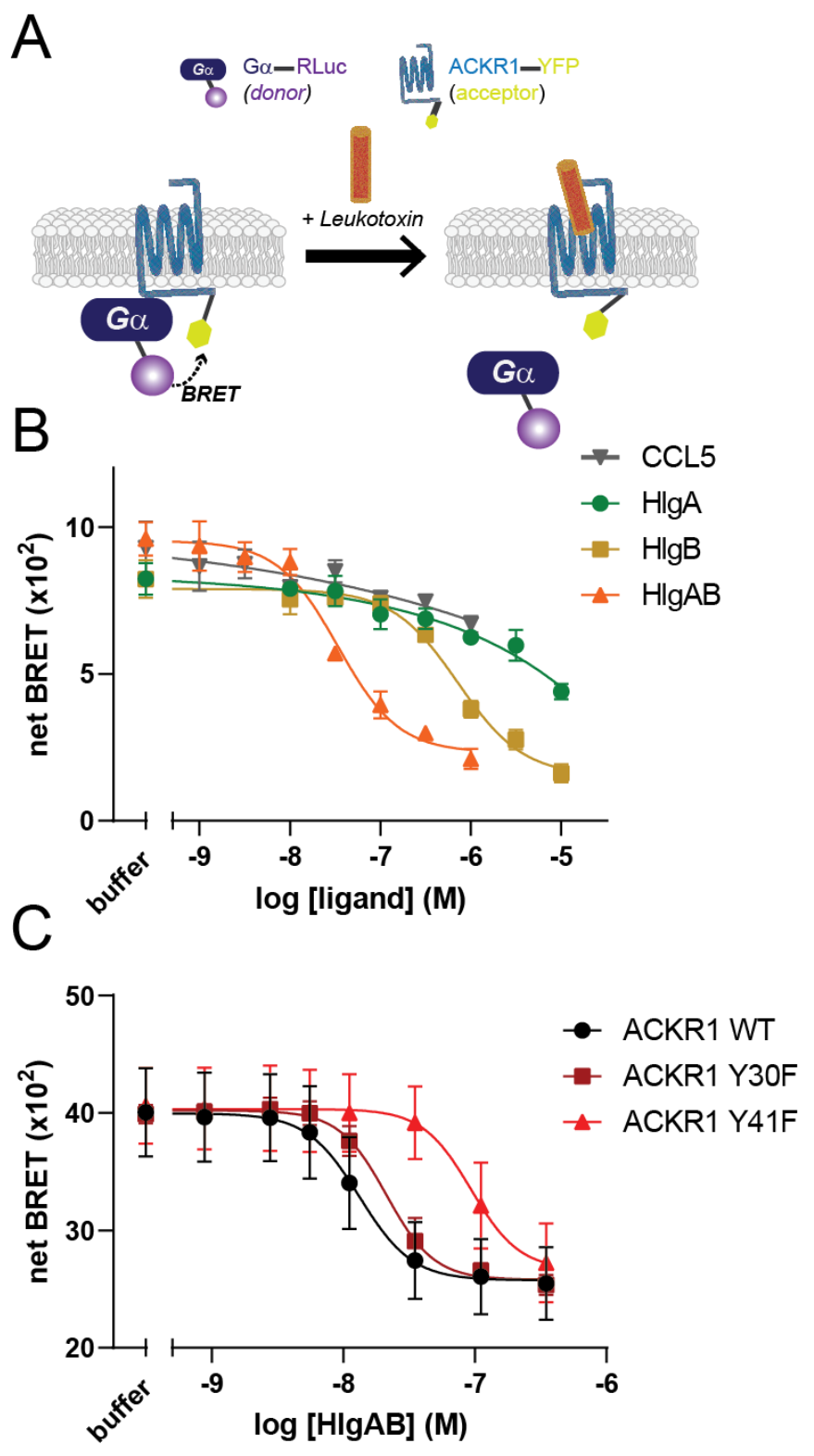

Figure 4 

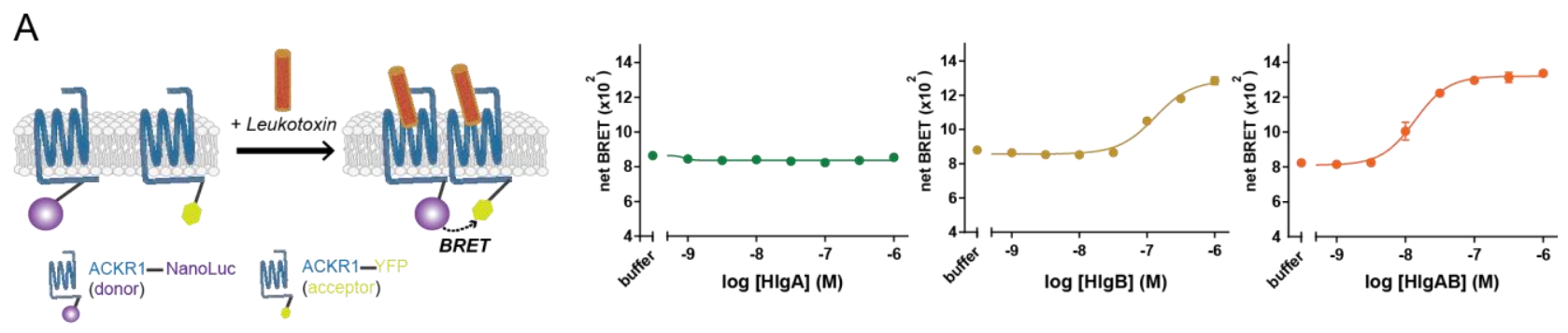

B
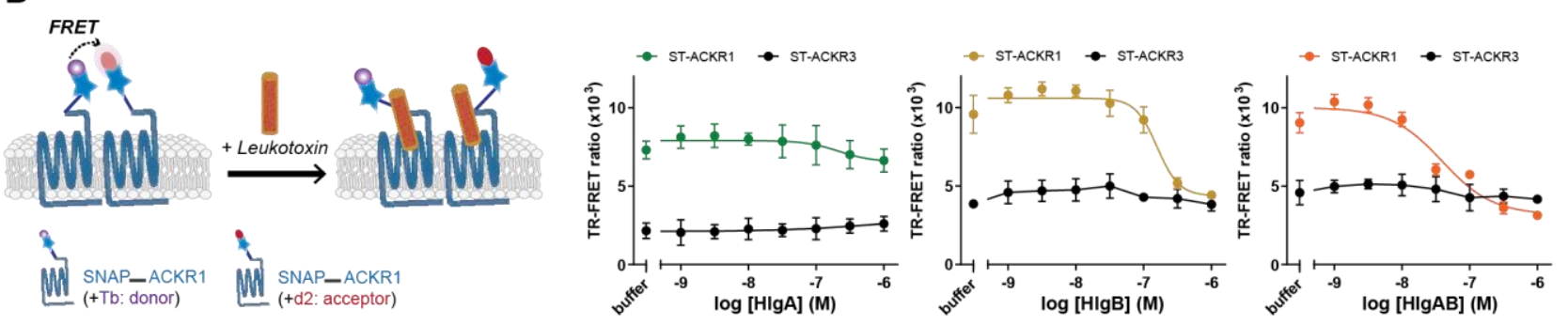

Figure 5 


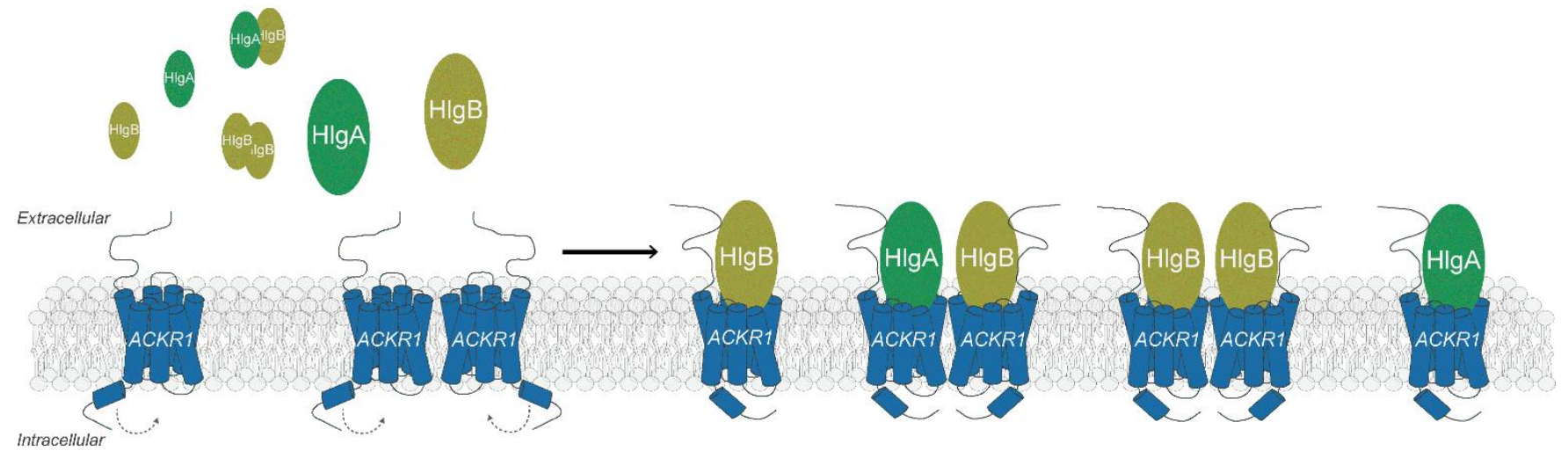

Figure 6 